\title{
Treatment Strategy for Oligometastatic Gastric Cancer: Brief Considerations
}

\author{
Daniele Lavacchi ${ }^{1}$ Elisa Giommoni ${ }^{1} \cdot$ Fabio Cianchi $^{2,3} \cdot$ Lorenzo Antonuzzo $^{1,3}$ \\ Accepted: 20 December 2021 \\ (c) The Author(s), under exclusive licence to Springer Science+Business Media, LLC, part of Springer Nature 2021
}

Despite recent advances in gastric cancer (GC) treatment, overall survival (OS) in metastatic disease remains approximately 1 year in patients fit for chemotherapy, and treatment options beyond the second-line are limited [1-3]. However, metastatic GC should be considered a heterogeneous group of diseases with different patterns of metastatic spread and specific molecular subclasses [1, 4]. While patients with high tumor burden and multi-organ metastases will never benefit from locoregional therapies, it is intriguing to contemplate that those with oligometastatic and chemosensitive disease may be candidates for a multimodal strategy $[4,5]$. In routinely clinical practice, GC patients who receive surgery for metastases account for around 5\% of all metastatic GC cases, but incidence varies across different study populations and definitions [6]. Although no definition of oligometastatic GC has yet achieved a prospective validation, inclusion criteria of arm B (limited metastatic) of the AIO-FLOT3 study with modifications according to the RENAISSANCE trial offer the best proof of concept for further investigation. Limited metastatic disease was defined as the presence of retroperitoneal lymph nodes and/or metastases to a single organ: $\leq 5$ metastases in case of liver involvement, unilateral or potentially resectable metastases in case of lung involvement. Diffuse peritoneal involvement was considered extensive metastatic disease, while localized peritoneal metastases (P1 score), unilateral or bilateral Krukenberg tumors, localized bone involvement within one radiation field, unilateral or bilateral adrenal gland metastases, and selected cases of extra-abdominal lymph node metastases

Lorenzo Antonuzzo

lorenzo.antonuzzo@unifi.it

1 Clinical Oncology Unit, Careggi University Hospital, Florence, Italy

2 Unit of Digestive Surgery, Careggi University Hospital, Florence, Italy

3 Department of Experimental and Clinical Medicine, University of Florence, Florence, Italy were included in oligometastatic disease [7, 8]. The AIOFLOT3 trial showed that patients with limited metastatic GC obtained an OS of 22.9 months (95\% CI, 16.5, upper level not achieved). Near $60 \%$ of patients underwent surgery and achieved a more favorable outcome, particularly for those with abdominal lymph node metastases only. However, this study was for exploratory purposes and, given the lack of randomization, did not provide a real estimate of the benefit of surgery compared to chemotherapy alone [7]. To address the open questions of the AIO-FLOT3 trial, Al-Batran and colleagues are conducting the phase III RENAISSANCE trial (NCT02578368) in which patients with oligometastatic disease receive FLOT for 4 cycles and are randomized to receive additional 4-8 cycles of FLOT or surgery followed by FLOT for $4-8$ cycles. Patients with chemorefractory disease dropped out without proceeding to randomization [8]. In recent years, FLOT regimen has been shown to increase the rate of pathological complete response and improve survival outcomes compared to ECF in perioperative setting, offering an acceptable safety profile as demonstrated by the phase III FLOT4-AIO trial and a large Italian observational study $[9,10]$.

The RENAISSANCE trial is expected to clarify the role of surgery in the treatment strategy of oligometastatic disease, probably confirming FLOT as the regimen of choice in this setting. However, what is the best chemotherapy regimen in this specific population remains to be investigated. New combinations are changing the first-line treatment of GC. At first, the addition of immune checkpoint inhibitors to standard chemotherapy improved OS compared to chemotherapy alone in specific subgroups of patients $[11,12]$. Second, the emerging new drugs directed against HER2 could increase response rate and long-lasting disease control also in patients with previously untreated GC [13]. Third, new genomic landscapes and therapeutic targets could rapidly found an application in the selection of the best frontline treatment for advanced disease [14-16]. 
While awaiting the results of the RENAISSANCE trial, the management of patients with oligometastatic GC deserves a multidisciplinary approach. Consistent with previous studies, frontline chemotherapy represents the major milestone in the treatment of oligometastatic GC. Surgery with curative intent should be considered in highly selected cases with good performance status, favorable tumor response to chemotherapy, and easily accessible lesions [4, $5,7,8,17]$. However, the goal of preserving the quality of life deserves great attention; thus, patients with chemorefractory disease, relevant comorbidities, or limited chances of curative resection should not receive surgery. Since palliative gastrectomy has not been shown to prolong survival, it should be offered as little as possible. Alongside the proper clinical selection, further efforts should be made to improve the molecular selection of patients who may benefit from the combined modality treatments $[1,4,5,7,18]$. Stimulating results in frontline tailored treatments are expected in the next few years. In this regard, the phase II PANGEA trial has shown remarkable results in patients managed with a personalized approach including chemotherapy in combination with targeted therapies individually optimized on the longitudinal molecular profile [14]. This aspect deserves further investigation in patients with potentially resectable metastatic GC.

\section{Declarations}

Conflict of Interest The authors declare no competing interests.

\section{References}

1. Smyth EC, Nilsson M, Grabsch HI, et al. Gastric cancer. Lancet. 2020;396(10251):635-48. https://doi.org/10.1016/S01406736(20)31288-5 (PMID: 32861308).

2. Roviello G, Fancelli S, Gatta Michelet MR, et al. TAS-102 in gastric cancer: development and perspectives of a new biochemically modulated fluoropyrimidine drug combination. Crit Rev Oncol Hematol. 2020;152:102987. https://doi.org/10.1016/j. critrevonc.2020.102987 (Epub 2020 May 21 PMID: 32485527).

3. Fanotto V, Cordio S, Pasquini G, et al. Prognostic factors in 868 advanced gastric cancer patients treated with second-line chemotherapy in the real world. Gastric Cancer. 2017;20(5):825-33. https://doi.org/10.1007/s10120-016-0681-6 (Epub 2016 Dec 27 PMID: 28028664).

4. Fornaro L, Spallanzani A, de Vita F, et al. Beyond the guidelines: the grey zones of the management of gastric cancer. Consensus Statements from the Gastric Cancer Italian Network (GAIN). Cancers (Basel). 2021;13(6):1304. Published 2021 Mar 15. https://doi.org/10.3390/cancers 13061304.

5. Salati M, Valeri N, Spallanzani A, et al. Oligometastatic gastric cancer: an emerging clinical entity with distinct therapeutic implications. Eur J Surg Oncol. 2019;45(8):1479-82. https:// doi.org/10.1016/j.ejso.2018.11.006 (Epub 2018 Nov 10 PMID: 30448343).

6. Carmona-Bayonas A, Jiménez-Fonseca P, Echavarria I, et al. Surgery for metastases for esophageal-gastric cancer in the real world: data from the AGAMENON national registry. Eur J Surg Oncol. 2018;44(8):1191-8. https://doi.org/10.1016/j.ejso.2018. 03.019 (Epub 2018 Mar 31 PMID: 29685755).

7. Al-Batran SE, Homann N, Pauligk C, et al. Effect of neoadjuvant chemotherapy followed by surgical resection on survival in patients with limited metastatic gastric or gastroesophageal junction cancer: the AIO-FLOT3 trial. JAMA Oncol. 2017;3(9):1237-44. https://doi.org/10.1001/jamaoncol.2017. 0515 .

8. Al-Batran SE, Goetze TO, Mueller DW, et al. The RENAISSANCE (AIO-FLOT5) trial: effect of chemotherapy alone vs. chemotherapy followed by surgical resection on survival and quality of life in patients with limited-metastatic adenocarcinoma of the stomach or esophagogastric junction - a phase III trial of the German AIO/CAO-V/CAOGI. BMC cancer vol. 17,1 893. 28 Dec. 2017. https://doi.org/10.1186/s12885-017-3918-9.

9. Al-Batran SE, Homann N, Pauligk C, et al. Perioperative chemotherapy with fluorouracil plus leucovorin, oxaliplatin, and docetaxel versus fluorouracil or capecitabine plus cisplatin and epirubicin for locally advanced, resectable gastric or gastro-oesophageal junction adenocarcinoma (FLOT4): a randomised, phase 2/3 trial. Lancet. 2019;393(10184):1948-57. https://doi.org/10.1016/S0140-6736(18)32557-1 (Epub 2019 Apr 11 PMID: 30982686).

10. Giommoni E, Lavacchi D, Tirino G, et al. Results of the observational prospective RealFLOT study. BMC Cancer. 2021;21(1):1086. https://doi.org/10.1186/s12885-021-087687.PMID:34625033;PMCID:PMC8499559.

11. Janjigian YY, Shitara K, Moehler M, et al. First-line nivolumab plus chemotherapy versus chemotherapy alone for advanced gastric, gastro-oesophageal junction, and oesophageal adenocarcinoma (CheckMate 649): a randomised, open-label, phase 3 trial. Lancet. 2021;398(10294):27-40. https://doi.org/10.1016/ S0140-6736(21)00797-2 (Epub 2021 Jun 5 PMID: 34102137).

12. Rodriquenz MG, Roviello G, D'Angelo A, et al. MSI and EBV positive gastric cancer's subgroups and their link with novel immunotherapy. J Clin Med. 2020;9(5):1427. https://doi.org/ 10.3390/jcm9051427.PMID:32403403;PMCID:PMC7291039.

13. Shitara K, Bang YJ, Iwasa S, et al. Trastuzumab deruxtecan in previously treated HER2-positive gastric cancer. N Engl J Med. 2020;382(25):2419-30. https://doi.org/10.1056/NEJMoa2004413 (Epub 2020 May 29 PMID: 32469182).

14. Catenacci DVT, Moya S, Lomnicki S, et al. Personalized antibodies for gastroesophageal adenocarcinoma (PANGEA): a phase II study evaluating an individualized treatment strategy for metastatic disease. Cancer Discov. 2021 Feb;11(2):308-25. https://doi.org/10.1158/2159-8290.CD-20-1408. Epub 2020 Nov 24. PMID: 33234578; PMCID: PMC7858231.

15. Danesi R, Fogli S, Indraccolo S, et al. Druggable targets meet oncogenic drivers: opportunities and limitations of target-based classification of tumors and the role of molecular tumor boards. ESMO Open. 2021 Apr;6(2):100040. https://doi.org/10.1016/j. esmoop.2020.100040. Epub 2021 Feb 2. PMID: 33540286; PMCID: PMC7859305.

16. Lavacchi D, Roviello G, D'Angelo A. Tumor-agnostic treatment for cancer: when how is better than where. Clin Drug Investig. 2020;40(6):519-27. https://doi.org/10.1007/s40261-020-009155 (PMID: 32307639).

17. Montagnani F, Crivelli F, Aprile G, et al. Long-term survival after liver metastasectomy in gastric cancer: systematic review and meta-analysis of prognostic factors. Cancer Treat Rev. 
2018;69:11-20. https://doi.org/10.1016/j.ctrv.2018.05.010 (Epub 2018 May 17 PMID: 29860024).

18. Fujitani K, Yang HK, Mizusawa J, et al. Gastrectomy plus chemotherapy versus chemotherapy alone for advanced gastric cancer with a single non-curable factor (REGATTA): a phase 3, randomised controlled trial. Lancet Oncol. 2016;17(3):309-18. https://doi.org/10.1016/S1470-2045(15)00553-7 (Epub 2016 Jan 26 PMID: 26822397).
Publisher's Note Springer Nature remains neutral with regard to jurisdictional claims in published maps and institutional affiliations. 